\title{
$4 \quad$ Kurz-, mittel- und langfristige Personalplanungen
}

\subsection{Darstellung}

Differenziert man Personalplanungsansätze nach der Fristigkeit des Planungszeitraums, so werden kurz- von mittel- und langfristigen Modellen unterschieden. Als Differenzierungskriterium dient somit der kalendarische Zeitbezug. Personalplanungsprobleme mit einem Horizont von bis zu drei Monaten werden dann häufig als kurz-, solche mit einem Horizont zwischen drei und zwölf Monaten oft als mittel- und solche, deren Planungshorizont über ein Jahr hinausreicht, werden häufig als langfristig bezeichnet. Selbstverständlich obliegt die konkrete Festlegung der Abgrenzung dem jeweiligen Entscheider und ist insofern nicht mit dem Anspruch auf generelle Gültigkeit verknüpft (zu einem anderen Abgrenzungsvorschlag vgl. z.B. Zahn 1989, Sp. 1085).

Viele Probleme der reinen Personaleinsatzplanung stellen kurzfristige Planungsprobleme dar, so z.B. wenn Arbeitskräfte einzelnen Maschinen oder Jobs zuzuordnen sind (Job Matching), wenn der Monatsdienstplan für das Pflegepersonal einer chirurgischen Klinik erstellt werden soll (Nurse Scheduling) oder wenn die Zuordnung von Busfahrern zu -linien eines städtischen Verkehrsbetriebes gesucht wird (Bus Driver Scheduling). Im Falle saisonaler (z.B. quartalsweiser), wiederkehrender Schwankungen des Personalbedarfs sind (z.B. in Warenhäusern oder in der Gastronomie) vielfach mittelfristige Personalbereitstellungsplanungen angebracht. Ein typisches Beispiel für langfristige Personalplanungen hingegen sind alle Fälle, in denen gleichzeitig Entscheidungen über das zukünftige Produktions- und das Investitionsprogramm sowie über die optimale Personalbereitstellung und -verwendung getroffen werden sollen (vgl. Spengler 1995, S. 9).

\section{2 Übungsaufgabe}

\section{Aufgabe 10}

Erläutern Sie neben dem kalendarischen Zeitbezug eine weitere Möglichkeit zur Abgrenzung von kurz-, mittel- und langfristigen Personalplanungen! 